\title{
Rapid method for the isolation of mammalian sperm DNA
}

\author{
Haotian Wu, Matthew K. de Gannes, Gianna Luchetti, and J. Richard Pilsner \\ Department of Environmental Health Sciences, School of Public Health and Health Sciences, University of \\ Massachusetts Amherst, Amherst, MA
}

\begin{abstract}
BioTechniques 58:293-300 (June 2015) doi 10.2144/000114280
Keywords: epigenetics; sperm; methods; nucleic acids; DNA purification; spermatozoa; DNA isolation; genetics
\end{abstract}

Supplementary material for this article is available at www.BioTechniques.com/article/114280.

The unique DNA packaging of spermatozoa renders them resistant to DNA isolation techniques used for somatic cells, requiring alternative methods that are slow and labor intensive. Here we present a rapid method for isolating high-quality sperm DNA. Isolated human sperm cells were homogenized with $0.2 \mathrm{~mm}$ steel beads for $5 \mathrm{~min}$ at room temperature in the presence of guanidine thiocyanate lysis buffer supplemented with 50 mM tris(2-carboxyethyl)phosphine (TCEP). Our method yielded >90\% high-quality DNA using 3 different commercially available silica-based spin columns. DNA yields did not differ between immediate isolation (2.84 $\pm 0.04 \mathrm{pg} / \mathrm{cell})$ and isolation after 2 weeks of homogenate storage at room temperature $(2.91 \pm 0.13 \mathrm{pg} / \mathrm{cell})$. DNA methylation analyses revealed similar methylation levels at both time points for three imprinted loci. Our protocol has many advantages: it is conducted at room temperature; lengthy proteinase $\mathrm{K}$ (ProK) digestions are eliminated; the reducing agent, TCEP, is odorless and stable at room temperature; nucleic acids are stabilized, allowing storage of homogenate; and it is adaptable for other mammalian species. Taken together, the benefits of our improved method have important implications for settings where sample processing constraints exist.

There is a growing interest in elucidating the role of sperm genetics and epigenetics on reproductive success and the life-course trajectory of health outcomes of subsequent generations. Recent genetic studies have shown a positive association between germline de novo mutations and paternal age (1-4). Aberrations in sperm DNA methylation of imprinted genes (5-7) and epigenome-wide dysregulation (8-10) have also been reported among men with infertility issues such as low sperm count and sperm quality. Moreover, compelling animal data indicate that the epigenome of sperm harbors a legacy of environmental exposures that can influence offspring phenotype (11-13).

Spermatogenesis requires extensive epigenetic reprogramming during the progression from diploid spermatogonia to haploid spermatozoa and involves stage- and testis-specific gene expression and mitotic and meiotic divisions (14,15). Extensive reorganization of chromatin structure occurs where $90 \%$ and $99 \%$ of histones are replaced by protamines in humans and mice, respectively $(16,17)$. During this protamine-histone transition, tight compaction of the sperm nucleus is achieved by the oxidation of cysteine- rich residues of protamines and the subsequent formation of disulfide bridges that link protamines together (18). This nuclear compaction is necessary for sperm motility and protection of the genome from oxidation within the female reproductive tract (19). Furthermore, the protamine-bound packaging of DNA precludes transcriptional activity and has been considered a nontraditional form of epigenetic regulation unique to sperm cells (20).

Their unique DNA packaging renders spermatozoa resistant to DNA isolation techniques used for somatic cells $(21,22)$. The development of

\section{METHOD SUMMARY}

Our optimized protocol for isolation of sperm DNA utilizes bead-based homogenization to facilitate sperm cell lysis in concert with an odorless reducing agent, tris(2-carboxyethyl)phosphine (TCEP), to dissociate disulfide bonds without the use of proteinase $\mathrm{K}$ (ProK). The procedure is conducted at room temperature, and the nucleic acids are sufficiently stabilized to allow storage of homogenate for future DNA isolation. After the homogenization step, DNA can be extracted by silica-based spin columns for a total processing time of 15-20 min. 
efficient methods for isolating DNA from mammalian sperm has been a gradual process. All existing protocols use a combination of three components to gain access to sperm DNA: (i) detergents and/or chaotropic salts to facilitate cell lysis; (ii) proteinase $\mathrm{K}$ (ProK) to digest nuclear proteins; and (iii) reducing agents to break disulfide bonds between protamines. One such widely adopted method for the isolation of mammalian sperm DNA uses an ionic detergent, sodium dodecyl sulfate (SDS), ProK, and either dithiothreitol (DTT) or 2-mercaptoethanol ( $\beta M E)$. After overnight incubation at $55^{\circ} \mathrm{C}$, DNA is isolated by ethanol precipitation (9) or silica-based spin columns (11). Other popular approaches utilize guanidine salts, such as guanidinium thiocyanate (GTC), as the cell lysis reagent. GTC is a chaotropic agent that disrupts cell membrane and organelles by solubilizing individual molecules or cellular structures, including separating nucleic acids from associated proteins (23). In addition, it is able to denature proteins, inactivate nucleases, and enhance ProK activity $(23,24)$. Bahnak et al. first reported a protocol incorporating GTC in a lysis buffer along with the ionic detergent, sarkosyl, and $\beta M E$ (21). However, this protocol required overnight incubations and time consuming $\mathrm{CsCl}$ ultracentrifugation for DNA isolation. More recently, this protocol has been modified to include ProK in the lysis buffer, which significantly reduced incubation time to $2 \mathrm{~h}$ and replaced lengthy $\mathrm{CsCl}$ ultracentrifugation with isopropanol precipitation of DNA, resulting in an $80 \%$ yield of sperm DNA $(24,25)$.

While the previous methods for sperm DNA isolation have progressed over time, they still have drawbacks. First the limited stability of DTT, $\beta M E$, and ProK in aqueous solutions at room temperature requires fresh preparation of lysis buffers and involves long incubations ranging from $2 \mathrm{~h}$ to overnight at $56^{\circ} \mathrm{C}(9,24-26)$. In addition, DTT and $\beta M E$ possess odors that may not be tolerated, especially in clinical settings. Finally, most protocols recover DNA from sperm lysate through ethanol precipitation, which increases processing time and may result in co-precipitation of proteins and/or ethanol carryover that may affect downstream applications.
Given the need for a simple and rapid protocol for sperm DNA isolation, we developed a novel approach for isolating high-quality sperm DNA. Our protocol incorporates a 5 min mechanical homogenization step in the presence of a guanidine-based lysis buffer and a thiol-free reducing agent, TCEP, to facilitate sperm cell lysis and dissociation of disulfide bonds without the use of ProK. Sperm lysate is then applied to silica-based columns for the isolation of high-quality DNA with $>90 \%$ yield. To further streamline our protocol, we use commercially-available reagents that are stable at room temperature. This method is likely to expedite genetic and epigenetic research of sperm in clinical settings as well as in other mammalian species.

\section{Methods}

Isolation of sperm cells

This study was approved by the Institutional Review Board at the University of Massachusetts Amherst (\#2014-2337). All participants gave written informed consent and were required to have at least $48 \mathrm{~h}$ of abstinence prior to each donation. Five healthy male participants each donated multiple whole ejaculate samples throughout the course of the study. To remove somatic cell contamination, sperm cells were isolated using a continuous one-step 90\% gradient (ART-2100 and ART-1006; Sage, Beverly, MA) per the manufacturer's protocol. Sperm pellets were washed once, re-suspended, counted on a hemocytometer using the average of eight grid areas, and visually inspected for somatic cell contamination. Isolated sperm from individuals ranged from 20 to 109 million cells.

\section{Cell lysis}

Reducing agents TCEP (final concentration: 10-50 mM) (\#77720; Pierce, Rockford, IL), DTT (final concentration: 150 mM) (\#V3151; Promega, Madison, $\mathrm{WI}$ ), or $\beta M E$ (final concentration: $2 \%$ ) were added to Buffer RLT (\#79216; Qiagen, Limburg, The Netherlands) to a final volume of $500 \mu \mathrm{L}$. Three different homogenization techniques were also evaluated: (i) sperm were pulse-vortexed in lysis buffer for 5 min, diluted 1:1 in nuclease free water, and then incubated with ProK (final concentration: $200 \mu \mathrm{g} / \mathrm{mL}$ ) at $56^{\circ} \mathrm{C}$ for $2 \mathrm{~h}$; (ii) sperm were pulse-vortexed in lysis buffer for $5 \mathrm{~min}$, and lysates were loaded onto Qiashredder columns (\#79656; Qiagen) and centrifuged for 2 min at maximum speed $(\geq 17,000 \times g)$; (iii) sperm cells were homogenized in the presence of lysis buffer and $0.1 \mathrm{~g}$ of $0.2 \mathrm{~mm}$ stainless steel beads (\#SSB02; Next Advance, Averill Park, NY) for 5 min on a Disruptor Genie (\#SI-238; Scientific Industries, Bohemia, NY). To ensure equal aliquots of sperm, resuspended sperm cells after gradient isolation were vortexed for $10 \mathrm{~s}$ between each aliquot as previously described (27).

\section{DNA isolation}

Sperm DNA was extracted with three different commercially-available kits using modified protocols:

AllPrep DNA/RNA Mini Kit (\#80204; Qiagen). Lysates were added to spin columns and centrifuged at 10,000 $x$ $g$ for $30 \mathrm{~s}$ to bind DNA. Subsequent washing steps followed the manufacturer's protocol. To elute, $50 \mu \mathrm{L}$ of Buffer EB (preheated to $70^{\circ} \mathrm{C}$ ) was added to the columns, which were incubated at room temperature for 3 min and then centrifuged for $1 \mathrm{~min}$ at maximum speed. This process was repeated twice for a total elution volume of $150 \mu \mathrm{L}$.

QIAamp DNAMiniKit(\#51304; Qiagen).

Lysates were combined with equal volumes of Buffer AL and $100 \%$ ethanol, loaded onto the spin columns, and the columns were centrifuged at $6000 \times$ $g$ for $1 \mathrm{~min}$ to bind DNA. Wash and elution steps followed the manufacturer's protocol, including 3 separate $200 \mu \mathrm{L}$ elutions to maximize yield.

Quick-gDNA MiniPrep (\#D3025; Zymo, Irvine, CA). DNA/RNA Shield (\#R1100; Zymo) and Quick gDNA Genomic Lysis Buffer (included in kit), were used for sperm lysis instead of Buffer RLT. Samples in the Genomic Lysis Buffer were loaded onto the columns, while samples in DNA/RNA Shield were combined with 3 volumes of Genomic Lysis Buffer before being loaded onto spin columns. Samples were centrifuged at 10,000 $\times g$ for $1 \mathrm{~min}$ to bind. Wash and elution steps followed the manufacturer's protocol for a final elution volume of $100 \mu \mathrm{L}$.

DNA yields and quality were determined using the Nanodrop 2000 
Spectrophotometer (\#E112352; Thermo Scientific, Somerset, NJ). A total of 350 ng of genomic DNA (gDNA) was resolved on a $0.7 \%$ agarose gel at $100 \mathrm{~V}$ for 45 $\mathrm{min}$, stained with $0.5 \mu \mathrm{g} / \mathrm{mL}$ ethidium bromide solution, and visualized on a BioDoc-It Imaging System (\#97-0172-01; UVP, Upland, CA). Given that haploid cells are expected to contain 3 pg DNA per cell, DNA yields were calculated as the observed yield/expected yield based on cell count. The full protocol for DNA isolation is provided in the Supplementary Material.

\section{RNA isolation}

Sperm cell lysate may be partitioned for the isolation of sperm RNA by adding 1:1 ratio of Qiazol and following the protocol of Goodrich et al. (28) starting at step 18 under section 3.1.

\section{DNA methylation analysis}

DNA methylation analyses of imprinted genes were performed on Sequenom's (San Diego, CA) MassARRAY platform, which uses RNA base-specific cleavage (MassCLEAVE) and matrix-assisted laser desorption/ionization time-of-flight mass spectrometry (MALDI-TOF MS) for quantitative DNA methylation analyses of PCR-amplified bisulfite-converted DNA (29). Briefly, $7.5 \mathrm{ng}$ of bisulfite converted DNA (\#D5002, EZ DNA Methylation Kit; Zymo Research) was amplified with reverse primers containing a T7-promoter tag. Primers and PCR conditions are provided in Supplementary Table S1. After treatment with shrimp alkaline phosphatase to remove unincorporated dNTPs, amplification products were subjected to in vitro transcription and T-specific cleavage and were then analyzed by MALDI-TOF MS. The C/T changes introduced by bisulfite treatment are reflected as $\mathrm{G} / \mathrm{A}$ changes on the T7-directed RNA transcript and result in a mass difference of $16 \mathrm{Da}$ for each $\mathrm{CpG}$ dinucleotide. The level of methylation for each $\mathrm{CpG}$ unit was quantified using EpiTYPER (Sequenom, San Diego, CA) software and methylation across loci was calculated as the average methylation of individual CpG units.

\section{Results and discussion}

To improve processing time and work flow, we exploited several areas in

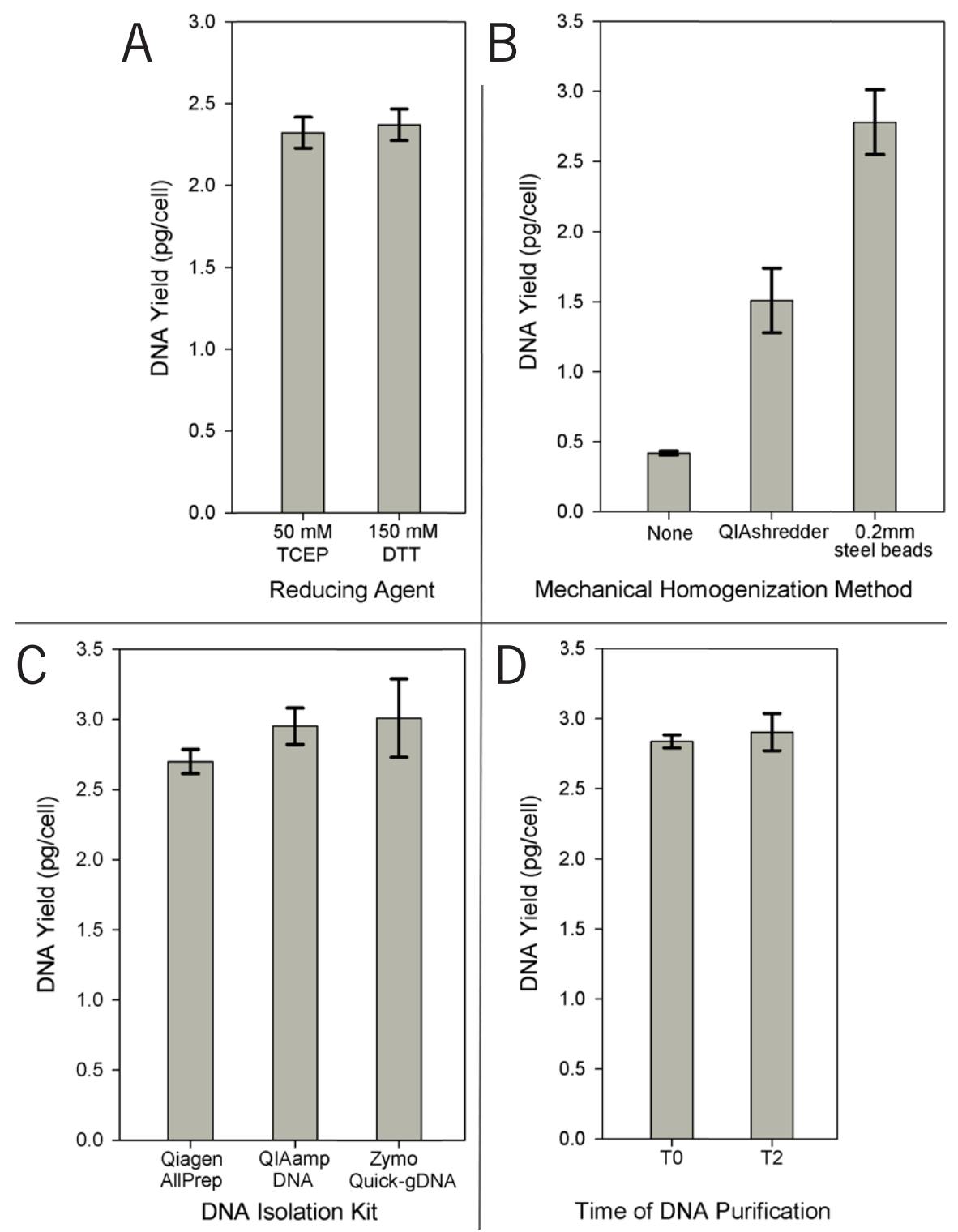

Figure 1. Utility of tris(2-carboxyethyl)phosphine (TCEP) as a reducing agent for the isolation of sperm DNA. (A) Mean ( \pm SD) DNA yield (pg/cell) of sperm cells treated with Buffer RLT, either $50 \mathrm{mM}$ TCEP or $150 \mathrm{mM}$ DTT, and proteinase $\mathrm{K}$ (ProK) for two hours at $56^{\circ} \mathrm{C}$. DNA was isolated from sperm cell lysate via AllPrep DNA columns. (B) Mean $( \pm$ SD) DNA yield (pg/cell) of sperm cells treated with Buffer RLT (50 mM TCEP) and the following homogenization methods: none; Qiashredder (as directed); or $0.1 \mathrm{~g}$ of $0.2 \mathrm{~mm}$ stainless steel beads. DNA was isolated from sperm cell lysate via AllPrep DNA columns. (C) Mean ( \pm SD) DNA yield (pg/cell) of sperm cells purified with Qiagen AllPrep, QIAmp Mini, or Zymo Quick-gDNA spin columns. Sperm cells were homogenized by $0.2 \mathrm{~mm}$ stainless steel beads in either Buffer RLT (AllPrep and DNA Mini) or Zymo gDNA lysis buffer (Quick gDNA), both supplemented with $50 \mathrm{mM}$ TCEP. (D) Mean ( \pm SD) DNA yield (pg/cell) of sperm cells homogenized by $0.2 \mathrm{~mm}$ stainless steel beads in the presence of Buffer RLT and 50 mM TCEP. DNA was isolated from sperm cell lysate via AllPrep spin columns processed immediately (TO) and after 2 weeks storage (T2) at room temperature $\left(22^{\circ} \mathrm{C}\right)$.

current protocols where considerable improvements could be achieved. Because recently published methods for sperm DNA isolation relied on userprepared GTC-based lysis buffer and ethanol precipitation, we reasoned that commercially-available GTC lysis buffers could offer an effective alternative to user-prepared lysis buffers as well as provide optimal DNA binding conditions for silica-based spin columns, thereby avoiding ethanol precipitation. Similar to the reported yield of $80 \%$ in a recent GTC-based method with ethanol precipitation (24), treatment of sperm cells with diluted Buffer RLT, $150 \mathrm{mM}$ DTT, and $200 \mu \mathrm{g} / \mathrm{mL}$ ProK for two hours followed by DNA isolation via AllPrep 
DNA columns resulted in a $79 \%$ yield $(2.37 \pm 10 \mathrm{pg} / \mathrm{cell})$ (Figure $1 \mathrm{~A})$. As an alternative, we examined the utility of TCEP, an odorless, room-temperature stable, thiol-free reducing agent primarily used for protein biochemistry. We found that $50 \mathrm{mM}$ TCEP resulted in no appreciable difference in DNA yield $(2.32 \pm 0.09 \mathrm{pg} / \mathrm{cell})$ compared with $150 \mathrm{mM}$ DTT (Figure 1A), indicating that TCEP is a viable alternative to thiolbased reducing agents for the isolation of sperm DNA.

With Buffer RLT's high concentration of GTC (2.8-4.0 M), we also reasoned that lengthy ProK digests could be circumvented if sperm cells are efficiently lysed. Therefore, we examined the utility of different mechanical homogenization techniques. QIAshredder, a columnbased mechanical homogenization system offered by Qiagen for the rapid homogenization of cells and tissues, resulted in better yield $(1.51 \pm 0.23 \mathrm{pg} /$ cell) compared with no homogenization (0.42 $\pm 0.01 \mathrm{pg} / \mathrm{cell}$ ) (Figure 1B), but was less effective compared with the $80 \%$ yields achieved by ProK digestions.

We next incorporated the novel homogenization method used for sperm RNA isolation developed by Krawetz and colleagues that utilizes $0.2 \mathrm{~mm}$ stainless steel beads (28). In the presence of Buffer RLT and $50 \mathrm{mM}$ TCEP, homogenization with $0.2 \mathrm{~mm}$ stainless steel beads resulted in $>90 \%$ yields (2.78 $\pm 0.23 \mathrm{pg} /$ cell) (Figure 1B). Given the interest in obtaining sperm DNA from abnormal sperm samples (e.g., those with low sperm counts), we determined the efficiency of our method was $>80 \%$ (i.e., 2.40 pg/cell) with $5 \times$ $10^{5}$ or more cells. Moreover, we tested our protocol down to $1 \times 10^{4}$ cells and were able to isolated sufficient DNA for bisulfite PCR of imprinted genes (data not shown).

Next, we evaluated the compatibility of the bead-based homogenization procedure with other reducing agents as well as other commerciallyavailable DNA column kits. Compared with $50 \mathrm{mM}$ TCEP, we found no appreciable differences in DNA yields for $150 \mathrm{mM}$ DTT and 2\% $\beta \mathrm{ME}$ using the same bead-based homogenization (data not shown). Furthermore, ProK digestion after homogenization did not increase DNA yield (data not shown),

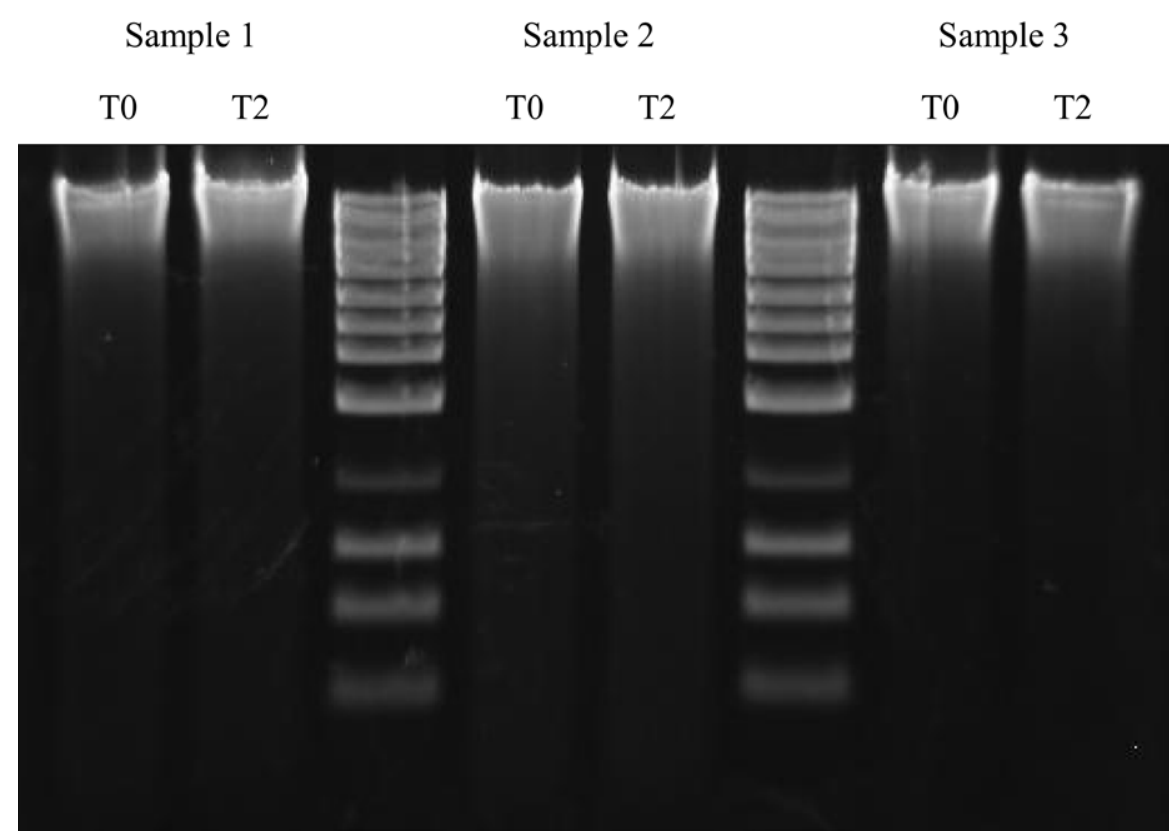

Figure 2. Electrophoresis of sperm DNA. Sperm DNA isolated from 3 individuals immediately (TO) and after 2 weeks of storage at room temperature (T2) on a $0.7 \%$ agarose gel.

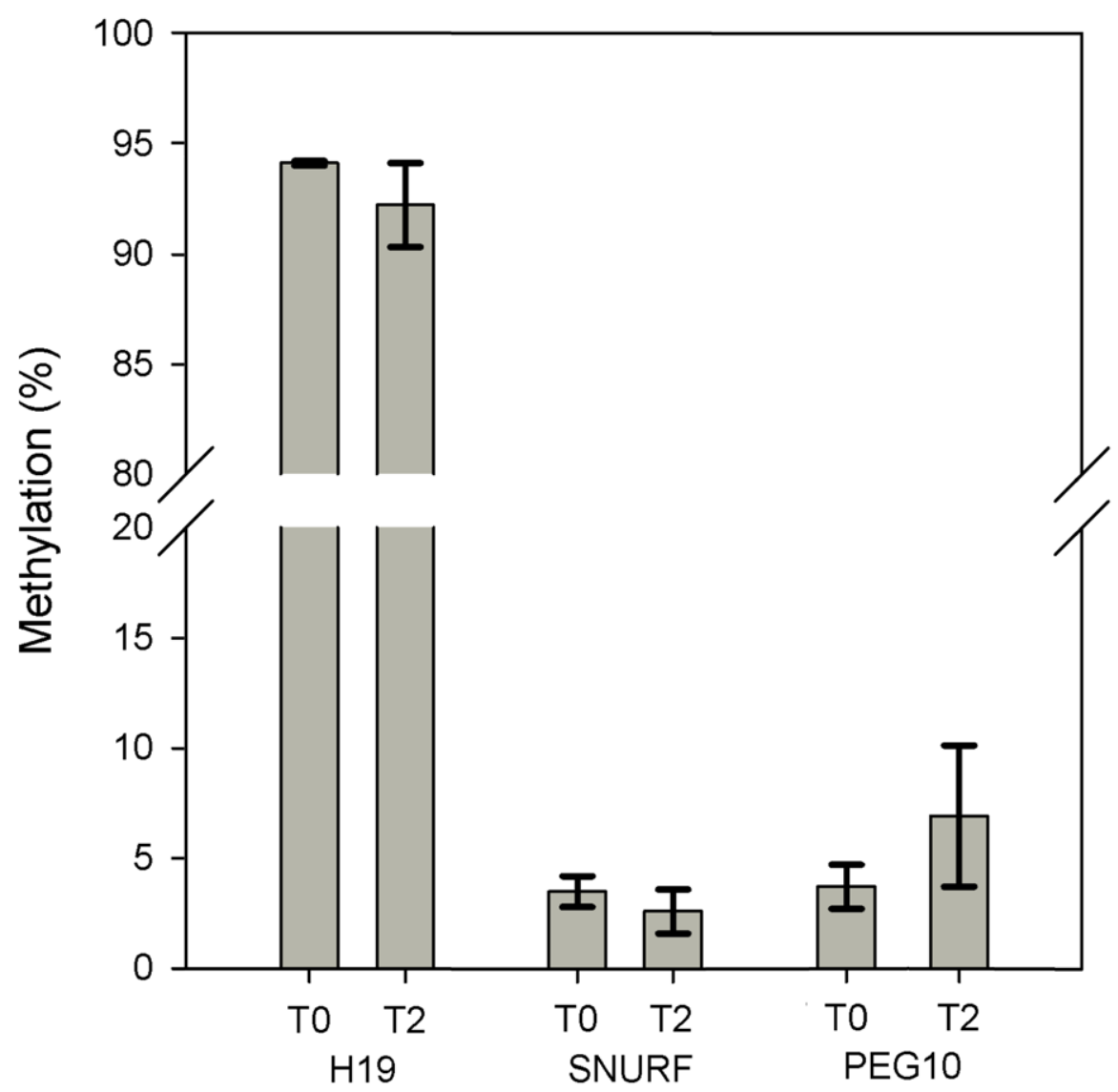

Figure 3. DNA methylation of three imprinted loci. Mean $( \pm S D)$ percentage DNA methylation of CpG sites within imprinted regions of SNURF, PEG10, and H19 using DNA isolated from sperm lysate immediately (TO) and after 2 weeks of storage at room temperature (T2). 


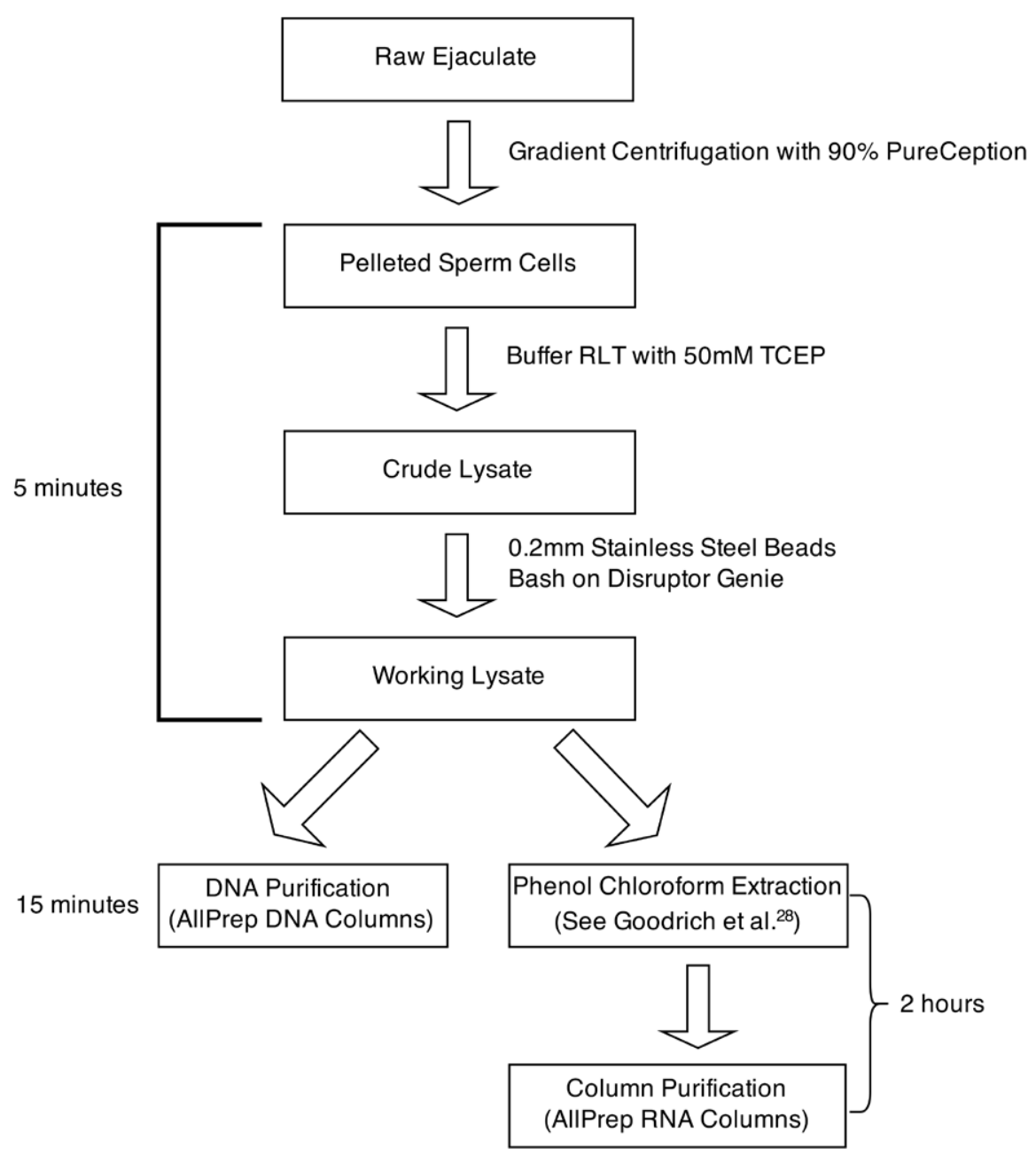

Figure 4. Schematic of sperm DNA isolation workflow. Sperm cells are isolated from ejaculate via gradient centrifugation with 90\% PureCeption. Pelleted sperm cells are then homogenized with Buffer RLT, $50 \mathrm{mM}$ tris(2-carboxyethyl)phosphine (TCEP), and $0.1 \mathrm{~g}$ of $0.2 \mathrm{~mm}$ stainless steel beads on a Disruptor Genie for 5 min. This produces a working lysate that is ready for DNA and RNA isolation.

indicating that lengthy ProK digestions are unnecessary. Therefore, this homogenization procedure circumvented the need for time-consuming ProK incubations. Compared with the AllPrep DNA columns, QIAamp DNA and Zymo's Quick-gDNA columns resulted in slightly higher overall yields $(2.70 \pm 0.09 \mathrm{pg} / \mathrm{cell}$ versus $2.95 \pm 0.13$ $\mathrm{pg} / \mathrm{cell}$ and $3.01 \pm 0.28 \mathrm{pg} / \mathrm{cell}$, respectively) (Figure 1C). Use of Zymo's QuickgDNA kit by replacing Buffer RLT with their genomic lysis buffer (contains $4 \mathrm{M}$ GTC) resulted in lower $A_{260} / A_{280}$ and $A_{260} /$ $A_{230}$ ratios compared with the other kits (data not shown).

We then aimed to test the stability and integrity of DNA in Buffer RLT and 50 mM TCEP after bead homogenization. Compared with immediate processing (T0), two weeks of storage (T2) at room temperature showed no decrease in DNA yield $(2.84 \pm 0.05 \mathrm{pg} /$ cell versus $2.91 \pm 0.13 \mathrm{pg} / \mathrm{cell}$, respectively) (Figure 1D). Sperm genomic DNA resolved on a $0.7 \%$ agarose gel revealed high molecular weight DNA that displayed no differences in integrity between samples processed immediately (TO) or after two weeks of storage (T2) at room temperature (Figure 2). Finally, to verify the feasibility of our protocol with downstream applications, we conducted DNA methylation analyses of three imprinted loci (SNURF, PEG10, H19). As expected for sperm DNA (30), differentially methylated regions (DMRs) of maternally expressed $\mathrm{H} 19$ displayed high levels of methylation, while DMRs of paternally expressed genes SNURF and PEG10 showed very low levels of methylation (Figure 3). Furthermore, we found no differences between samples that were processed immediately (TO) and after two weeks of storage (T2) at room temperature for DNA methylation levels of $H 19$ (TO: $94.1 \% \pm 0.1 \%$ and T2: $92.2 \% \pm 1.9 \%$ ), SNURF (T0: $3.5 \%$ $\pm 0.7 \%$ and $\mathrm{T} 2: 2.6 \% \pm 1 \%$ ), and PEG10 (TO: $3.7 \% \pm 1.8 \%$ and T2: $6.9 \% \pm 3.2 \%$ ) (Figure 3).

The use of the commerciallyavailable GTC Iysis buffers streamlined our protocol by eliminating the preparation of lysis buffers and allowing for optimal binding conditions for standard silica column-based DNA kits. We recommend Qiagen's RLT lysis buffer and columns because (i) DNA yields and $\mathrm{A}_{260} / \mathrm{A}_{280}$ ratios were consistently greater than $90 \%$ and 1.80 , respectively, and (ii) sperm cell lysates can be partitioned for sperm RNA purification using a previously published protocol (28). In addition to the DNA columns from the AllPrep DNA/RNA kit, we found that the QIAamp DNA Mini kit and Zymo's Quick-gDNA MiniPrep kit can also be adapted to use the RLT-based lysate with similar total yields. However, there are drawbacks to both the QIAamp and Zymo kits. The QIAamp DNA Mini Kit achieves similar total yield compared with AllPrep DNA/ RNA kit, but it requires a higher elution volume for maximum yield (600 $\mu \mathrm{L})$, thereby lowering final concentrations. This may not be desirable when there is limited starting material. While Zymo's Quick gDNA kit had the highest yield, it must be noted that the $A_{260} / A_{280}$ and $A_{260} / A_{230}$ ratios were consistently low, $<1.8$ and $<1.0$, respectively, suggesting Iow purity of DNA due to protein and/or GTC carryover.

Clinical research can present its own set of unique challenges. In studies where semen samples are processed at an in vitro fertilization laboratory, organic thiols may not be permissible due to their strong sulfur odor. TCEP irreversibly reduces disulfides and is reported to be more effective at reducing disulfides than DTT below $\mathrm{pH}$ 8.0 (31), which represents the typical $\mathrm{pH}$ of GTC lysis buffers (e.g., Qiagen $\mathrm{RLT}$ is $\mathrm{pH}$ 7.0). Moreover, while DTT and $\beta M E$ readily undergo atmospheric oxidation, TCEP is resistant to oxidation, allowing for room temperature storage in aqueous solutions. Our results show no appreciable difference in DNA yield 
between TCEP and thiol reductants and support previous data that TCEP is equally as effective as DTT at lysing sperm cells and may provide an effective substitute for organic thiols (27). Taken together, TCEP offers an odor-free and room-temperature stable alternative for the reduction of disulfide bonds, thereby making it our preferred reducing agent for DNA isolation of sperm cells.

The workflow of our protocol presents several practical advantages when isolating sperm DNA. First, our protocol provides flexibility, such that the stability of DNA after homogenization allows for the convenient storage of lysates for future downstream DNA isolation. Secondly, our protocol is streamlined by incorporating commercially available reagents that are stable at room temperature and eliminating the need for lengthy ProK digestions. These first two points have important implications, especially in regard to research in clinical settings where technician time may be limited. Moreover, the potential for lysate storage prior to DNA isolation and the use of commercially available reagents minimizes potential batch effects when conducting large epidemiologic studies. Our protocol is also amenable to partitioning lysate for RNA purification from the same sample. For example, given $500 \mu \mathrm{L}$ of lysate that contains $3 \times 10^{7}$ sperm, $100 \mu \mathrm{L}$ of lysate is expected to provide, at $90 \%$ yield, approximately $16 \mu \mathrm{g}$ of DNA with our protocol. The remaining $400 \mu \mathrm{L}$ of lysate can then be used for the isolation of RNA, which is present in extremely low quantities in sperm (50 fg/cell) (28). By incorporating the sperm RNA isolation method of Goodrich et al. (28), our theoretical example is estimated to provide $1.2 \mu \mathrm{g}$ of RNA. These yields provide ample nucleic acids for many downstream applications, including next-generation sequencing techniques. Additionally, our protocol is optimized for silica-based spin columns, thereby avoiding ethanol precipitation procedures. Lastly, our protocol can be extended for isolation of sperm DNA in other mammalian species. For example, our method is equally effective for isolating sperm DNA from mice (data not shown).
In conclusion, our optimized $5 \mathrm{~min}$ room-temperature homogenization protocol results in $>90 \%$ yield of highquality sperm DNA by utilizing steel beads to facilitate sperm cell lysis, in concert with the odorless reducing agent TCEP to dissociate disulfide bonds without the use of ProK. After homogenization, in lieu of lengthy ethanol precipitation, DNA can be extracted by user-preferred silica-based spin columns for a total processing time of 15-20 min. Our protocol also stabilizes nucleic acids, allowing for optional storage of homogenate for DNA isolation at a later date. A schematic of the DNA isolation method is shown in Figure 4, and a full protocol can be found in the Supplementary Material. Our improved method has important practical advantages for research in clinical settings where sample processing constraints likely exist.

\section{Author Contributions}

H.W., M.D.G., G.L., and J.R.P. contributed to the development and

\section{STOP BURYING YOUR HEAD IN THE SAND.}

\section{YOUR CELLLS COULD BE CONTAMINATED WITH MYCOPLASMA.}

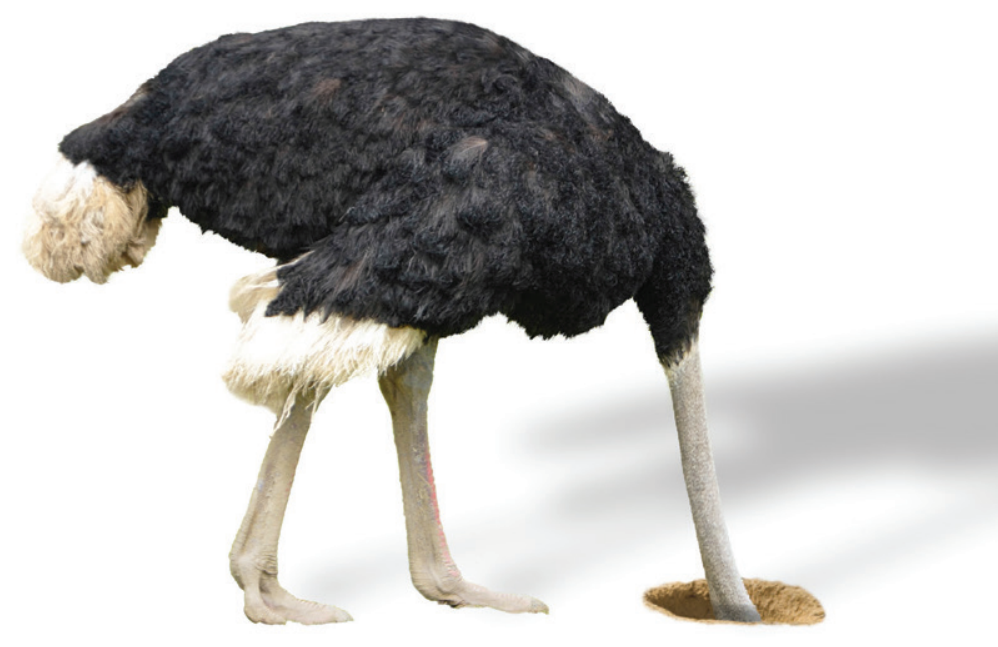

Mycoplasma contamination is a major concern for cell biologists. Although invisible, mycoplasmas can cause disastrous effects on eukaryotic cells as they can alter every cellular parameter from proliferation to virus susceptibility and production, significantly impacting the interpretation of biological results. Protect your cells with advanced and proven mycoplasma defense from InvivoGen.

The ideal tools to keep your cells mycoplasma-free:

$\Rightarrow$ Detect: PlasmoTest ${ }^{\mathrm{TM}}$

$\Rightarrow$ Eradicate: Plasmocin ${ }^{\mathrm{TM}}-$ Plasmocure $^{\mathrm{TM}}$

$\Rightarrow$ Prevent: Normocin ${ }^{\mathrm{TM}}$ - Primocin $^{\mathrm{TM}}$ 
execution of the experiments as well as the writing and editing of the manuscript. H.W. and J.R.P. contributed to the analysis of the experiments.

\section{Acknowledgments}

We gratefully acknowledge former Pilsner lab members Maggie Kwong and Justin English, who have contributed to this project. This work was supported by National Institute of Environmental Health Sciences grant K22-ES023085-01. This paper is subject to the NIH Public Access Policy.

\section{Competing interests}

The authors declare no competing interests.

\section{References}

1. Kong, A., M.L. Frigge, G. Masson, S. Besenbacher, P. Sulem, G. Magnusson, S.A. Gudjonsson, A. Sigurdsson, et al. 2012. Rate of de novo mutations and the importance of father's age to disease risk. Nature 488:471-475.

2. O'Roak, B.J., L. Vives, S. Girirajan, E. Karakoc, N. Krumm, B.P. Coe, R. Levy, A. Ko, et al. 2012. Sporadic autism exomes reveal a highly interconnected protein network of de novo mutations. Nature 485:246-250.

3. Neale, B.M., Y. Kou, L. Liu, A. Ma'ayan, K.E. Samocha, A. Sabo, C.F. Lin, C. Stevens, et al. 2012. Patterns and rates of exonic de novo mutations in autism spectrum disorders. Nature 485:242-245.

4. Sanders, S.J., M.T. Murtha, A.R. Gupta, J.D. Murdoch, M.J. Raubeson, A.J. Willsey, A.G. Ercan-Sencicek, N.M. DiLullo, et al. 2012. De novo mutations revealed by wholeexome sequencing are strongly associated with autism. Nature 485:237-241.

5. Kobayashi, H., A. Sato, E. Otsu, H. Hiura, C. Tomatsu, T. Utsunomiya, H. Sasaki, N. Yaegashi, and T. Arima. 2007. Aberrant DNA methylation of imprinted loci in sperm from oligospermic patients. Hum. Mol. Genet. 16:2542-2551.

6. Marques, C.J., F. Carvalho, M. Sousa, and A. Barros. 2004. Genomic imprinting in disruptive spermatogenesis. Lancet 363:17001702.

7. Marques, C.J., P. Costa, B. Vaz, F. Carvalho, S. Fernandes, A. Barros, and M. Sousa. 2008. Abnormal methylation of imprinted genes in human sperm is associated with oligozoospermia. Mol. Hum. Reprod. 14:67-74.

8. Hammoud, S.S., D.A. Nix, A.O. Hammoud, M. Gibson, B.R. Cairns, and D.T. Carrell. 2011. Genome-wide analysis identifies changes in histone retention and epigenetic modifications at developmental and imprinted gene loci in the sperm of infertile men. Hum. Reprod. 26:2558-2569.

9. Pacheco, S.E., E.A. Houseman, B.C. Christensen, C.J. Marsit, K.T. Kelsey, M. Sigman, and K. Boekelheide. 2011. Integrative DNA methylation and gene expression analyses identify DNA packaging and epigenetic regulatory genes associated with low motility sperm. PLoS ONE 6:e20280.

10. Houshdaran, S., V.K. Cortessis, K. Siegmund, A. Yang, P.W. Laird, and R.Z. Sokol. 2007. Widespread epigenetic abnormalities suggest a broad DNA methylation erasure defect in abnormal human sperm. PLoS ONE 2:e1289.

11. Radford, E.J., M. Ito, H. Shi, J.A. Corish, K. Yamazawa, E. Isganaitis, S. Seisenberger, T.A. Hore, et al. 2014. In utero effects. In utero undernourishment perturbs the adult sperm methylome and intergenerational metabolism. Science 345:1255903.

12. Lambrot, R., C. Xu, S. Saint-Phar, G. Chountalos, T. Cohen, M. Paquet, M. Suderman, M. Hallett, and S. Kimmins. 2013. Low paternal dietary folate alters the mouse sperm epigenome and is associated with negative pregnancy outcomes. Nat Commun. 4:2889.

13. Wei, Y., C.R. Yang, Y.P. Wei, Z.A. Zhao, Y. Hou, H. Schatten, and Q.Y. Sun. 2014. Paternally induced transgenerational inheritance of susceptibility to diabetes in mammals. Proc. Natl. Acad. Sci. USA 111:1873-1878.

14. Godmann, M., R. Lambrot, and S. Kimmins. 2009. The dynamic epigenetic program in male germ cells: Its role in spermatogenesis, testis cancer, and its response to the environment. Microsc. Res. Tech. 72:603-619.

15. Carrell, D.T. 2012. Epigenetics of the male gamete. Fertil. Steril. 97:267-274.

16. Hammoud, S.S., D.A. Nix, H. Zhang, J. Purwar, D.T. Carrell, and B.R. Cairns. 2009. Distinctive chromatin in human sperm packages genes for embryo development. Nature 460:473-478.

17. Brykczynska, U., M. Hisano, S. Erkek, L. Ramos, E.J. Oakeley, T.C. Roloff, C. Beisel, D. Schubeler, et al. 2010. Repressive and active histone methylation mark distinct promoters in human and mouse spermatozoa. Nat. Struct. Mol. Biol. 17:679-687.

18. Balhorn, R. 2007. The protamine family of sperm nuclear proteins. Genome Biol. 8:227.

19. Oliva, R. 2006. Protamines and male infertility. Hum. Reprod. Update 12:417-435.

20. Carrell, D.T. 2012. Epigenetics of the male gamete. Fertil. Steril. 97:267-274.

21. Bahnak, B.R., Q.Y. Wu, L. Coulombel, L. Drouet, D. Kerbiriou-Nabias, and D. Meyer. 1988. A simple and efficient method for isolating high molecular weight DNA from mammalian sperm. Nucleic Acids Res. 16:1208.

22. Shiurba, R. and S. Nandi. 1979. Isolation and characterization of germ line DNA from mouse sperm. Proc. Natl. Acad. Sci. USA 76:3947-3951.

23. Sawyer, W.H. and J. Puckridge. 1973. The dissociation of proteins by chaotropic salts. J. Biol. Chem. 248:8429-8433.

24. Griffin, J. 2013. Methods of sperm DNA extraction for genetic and epigenetic studies. Methods Mol. Biol. 927:379-384.

25. Hossain, A.M., B. Rizk, A. Behzadian, and I.H. Thorneycroft. 1997. Modified guanidinium thiocyanate method for human sperm DNA isolation. Mol. Hum. Reprod. 3:953-956.

26. Montjean, D., C. Ravel, M. Benkhalifa, P. Cohen-Bacrie, I. Berthaut, A. Bashamboo, and K. McElreavey. 2013. Methylation changes in mature sperm deoxyribonucleic acid from oligozoospermic men: assessment of genetic variants and assisted reproductive technology outcome. Fertil Steril. 100:12411247.

27. Bienvenue, J.M., N. Duncalf, D. Marchiarullo, J.P. Ferrance, and J.P. Landers. 2006. Microchip-based cell lysis and DNA extraction from sperm cells for application to forensic analysis. J. Forensic Sci. 51:266-273.

28. Goodrich, R.J., E. Anton, and S.A. Krawetz. 2013. Isolating mRNA and small noncoding RNAs from human sperm. Methods Mol. Biol. 927:385-396.

29. van den Boom, D. and M. Ehrich. 2009. Mass spectrometric analysis of cytosine methylation by base-specific cleavage and primer extension methods. Methods Mol. Biol. 507:207-227.

30. Filipponi, D. and R. Feil. 2009. Perturbation of genomic imprinting in oligozoospermia. Epigenetics 4:27-30.

31. Han, J.C. and G.Y. Han. 1994. A procedure for quantitative determination of tris(2-carboxyethyl)phosphine, an odorless reducing agent more stable and effective than dithiothreitol. Anal. Biochem. 220:5-10.

Received 27 January 2015; accepted 11 March 2015.

Address correspondence to J. Richard Pilsner, Department of Environmental Health Sciences, School of Public Health and Health Sciences, University of Massachusetts Amherst, Amherst, MA. E-mail: rpilsner@umass.edu

To purchase reprints of this article, contact: biotechniques@fosterprinting.com 